\title{
Multi-Service Data Dissemination for Space-based Augmentation Systems
}

\author{
Mariano Vergara, Felix Antreich, Gianluigi Liva, Balazs Matuz \\ Institute of Communications and Navigation \\ German Aerospace Center (DLR) \\ Wessling, Germany, 82234 \\ \{mariano.vergara, felix.antreich, gianluigi.liva, balazs.matuz $\} @$ dlr.de
}

\begin{abstract}
New SBAS (Satellite Based Augmentation System) signals could include the provision of new additional services alongside the defined SBAS L1/L5 aeronautical service [1], if additional power becomes available. In this paper we present a solution that efficiently makes use of the additional available power in order to increase the overall data rate of a new multiservice SBAS signal. These new SBAS signals will be backward compatible. The high power efficiency of the proposed scheme is guaranteed by a variation of the interplex scheme that is characterized by a variable envelope signal constellation. A coding scheme based on generalized low density parity check (LDPC) codes ensures that service requirements can be met with a lower SNR (Signal-to-Noise Ratio).
\end{abstract}

\section{Table of Contents}

1 InTRODUCTION.............................. 1

2 Signal MODEL ............................

3 Channel Code Design for the Short

BLOCK LENGTH REGIME ................... 5

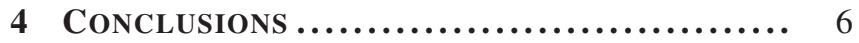

REFERENCES .............................. 6

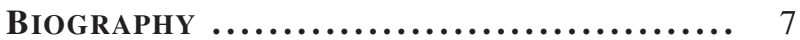

\section{INTRODUCTION}

The challenge posed by the design of new SBAS (Satellite Based Augmentation System) [1] signals is that the new signals must guarantee backward compatibility for legacy aeronautical users. Additional new multi-service SBAS signals must provide higher data rates in order to incorporate additional services, e.g. maritime, precise point positioning (PPP), authentication, or alert messaging service. At the same time, they must be particularly power efficient, as the power available on a satellite is a limiting factor in satellite communications and navigation, where receivers have small antenna gains. Additionally, a sound design must also allow certain flexibility in the allocation of resources among the new services, in order to follow future market trends and user demands.

The scheme we chose in this study to multiplex other services together with the SBAS L1/L5 aeronautical service is the interplex. The interplex is a multiplexing/mapping scheme that maps several signal components onto one carrier frequency [2]. Compared to other phase-shift-keyed/phasemodulated (PSK/PM) multichannel systems, the interplex is characterized by higher power efficiency, provided the number of signal components is not too large [2]. As other PSK/PM signals, the interplex signal possesses a constant

978-1-4673-1813-6/13/\$31.00@2013 IEEE. envelope constellation. A constant envelope constellation is very helpful in minimizing the non-linear distortions caused by the High Power Amplifier (HPA). This allows to drive the HPA at saturation, where the power efficiency is maximal. Although a constant envelope signal suffers little of the nonlinear distortion of the HPA, a constant envelope constellation has a price. This price comes in form of additional intermodulation (IM) products that are not used at the receiver, and thus they constitute a source of power inefficiency.

In this work, the interplex scheme is modified such that the additional IM products are scaled and the signal constellation is not exactly constant envelope. The advantage of this scheme allows to adapt the interplex scheme by designing a signal that is tailored to the available HPA. Indeed, the amount of power invested in the IM products can be chosen in such a way to reduce the non-linear distortions caused by the HPA within reasonable margin and on the other hand without an excessively large IM power.

The second element of novelty that we propose in this paper is an innovative coding scheme that encodes the signal components in a joint fashion by developing generalized low density parity check (LDPC) codes [3]. More specifically, we introduce a framework that allows jointly encoding and decoding the legacy signal, the main signal component, and the additional components while simultaneously keeping the legacy signal (aeronautical service) protected by the code defined for the baseline L1/L5 SBAS service [1]. In this work we present an example of such a coding scheme based on extended Hamming codes instead of convolutional codes as defined in [1].

\section{Signal MODEL}

In this section we present the novel interplex scheme, we show how this technique efficiently can make use of the available power, and we describe the HPA model that is used in this study.

\section{Scalable interplex}

An $N$-channel interplex [2] signal is a PSK/PM signal

$$
x_{N}(t)=\sin \left(2 \pi f_{c} t+\Theta(t)\right),
$$

in which the phase modulation is

$$
\Theta(t)=\left[\theta_{1}+\sum_{n=2}^{N} \theta_{n} s_{n}(t)\right] s_{1}(t),
$$

where $f_{c}$ denotes the carrier frequency and $\theta_{n}$ are the modulation (or interplex) angles. The signal components $s_{n}(t), n=$ $1, \ldots, N$ are direct sequence code division multiple access 
(DS-CDMA) signals, spread by Gold sequences [4]. We define the vectors:

$$
\begin{aligned}
\mathbf{b}^{n} & =\left[b_{0}^{n}, \ldots b_{K-1}^{n}\right]^{T} \in\{-1,1\}^{K \times 1} \\
\mathbf{c}^{n} & =\left[c_{0}^{n}, \ldots c_{G-1}^{n}\right]^{T} \in\{-1,1\}^{G \times 1}
\end{aligned}
$$

to represent the sequence of $K$ data symbols and the spreading code respectively, transmitted on the $n$-th signal component. $G \in \mathbb{N}$ is the processing gain of the DS-CDMA transmission. The signal components can be expressed as

$$
\mathbf{s}_{n}=\mathbf{b}^{n} \otimes \mathbf{c}^{n} \in\{-1,1\}^{K G \times 1}
$$

where $\otimes$ indicates the Kronecker product. We use the convention of calling $s_{1}(t)$ primary or main component, since it corresponds to the baseline SBAS L1/L5 signal; $s_{n}, \forall n \neq 1$ are denoted by secondary components and are the additional components that are used to convey new services. The signal components are normalized in order to have unitary power, i.e. $\left\|\mathbf{c}^{n}\right\|_{2}^{2}=G, \forall n$. In the following, the quantity $\theta_{1}$ in (2) is chosen equal to $\frac{\pi}{2}$, as this suppresses the carrier and good part of the IM products [2]. Moreover, we assume that all secondary components have the same power, so $\theta_{n}=\theta$, $\forall n \neq 1$. In this paper we propose a modification of the interplex scheme (1) with a variable constellation. Since the focus of this work is the shaping of the signal constellation, we will not consider the state transitions, as we did in [5]. This justifies our signal model (4) without pulse shape.

The (baseband) signal constellation of an $N$-channel interplex can be written as:

$$
\begin{aligned}
\mathbf{x}_{N}(\theta)=\mathbf{s}_{1}( & \cos \theta)^{N-1}+\mathbf{m}^{I}(\theta) \\
& +j\left(-\sum_{n=2}^{N} \mathbf{s}_{n}(\cos \theta)^{N-2} \sin \theta+\mathbf{m}^{Q}(\theta)\right)
\end{aligned}
$$

where $\mathbf{m}^{I}(\theta)$ and $\mathbf{m}^{Q}(\theta)$ indicate the vectors containing the IM terms, which are introduced to make the instantaneous power of the interplex constellation constant (5). The vectors $\mathbf{m}^{I}(\theta)$ and $\mathbf{m}^{Q}(\theta)$ have the same dimensions as $\mathbf{s}_{n}$. In order to define $\mathbf{m}^{I}(\theta)$ and $\mathbf{m}^{Q}(\theta)$, we introduce the following quantities:

$\mathbf{Z}_{n}^{N-1} \in\{-1,1\}^{K G \times 1}$ : vector containing the sum of all Hadamard products denoted by $\odot$, containing $n$ factors, obtainable from all possible $n$-combinations of the set $\left\{\mathbf{s}_{2}, \ldots, \mathbf{s}_{N}\right\}$. Example for $n=2: \mathbf{Z}_{2}^{N-1}=\mathbf{s}_{2} \odot \mathbf{s}_{3}+\mathbf{s}_{2} \odot \mathbf{s}_{4}+$ $\cdots+\mathbf{s}_{2} \odot \mathbf{s}_{N}+\mathbf{s}_{3} \odot \mathbf{s}_{4}+\ldots$

${ }^{1} \mathbf{Z}_{n}^{N-1} \in\{-1,1\}^{K G \times 1}$ : vector containing the sum of all Hadamard products, containing $n+1$ factors, obtainable from all possible $n$-combinations of the set $\left\{\mathbf{s}_{2}, \ldots, \mathbf{s}_{N}\right\}$, including the factor $\mathbf{s}_{1}$ in each $n$-combination. Example for $n=2$ : ${ }^{1} \mathbf{Z}_{2}^{N-1}=\mathbf{s}_{1} \odot \mathbf{s}_{2} \odot \mathbf{s}_{3}+\mathbf{s}_{1} \odot \mathbf{s}_{2} \odot \mathbf{s}_{4}+\cdots+\mathbf{s}_{1} \odot \mathbf{s}_{2} \odot$ $\mathbf{s}_{N}+\mathbf{s}_{1} \odot \mathbf{s}_{3} \odot \mathbf{s}_{4}+\ldots$.

The values of $\mathbf{m}^{I}(\theta)$ and $\mathbf{m}^{Q}(\theta)$ can be expressed for any value of $N$ as:

$$
\begin{aligned}
& \mathbf{m}^{I}(\theta)= \begin{cases}\mathbf{0}, & N<3 \\
\sum_{\substack{n=2 \\
n \text { even }}}^{N-1} \operatorname{sign}\left[\sin \left(2 \pi \frac{\mathrm{n}-2}{4}-\frac{\pi}{4}\right)\right] \times & \\
{ }^{1} \mathbf{Z}_{n}^{N-1}(\sin \theta)^{n}(\cos \theta)^{N-n-1}, & N \geq 3\end{cases}
\end{aligned}
$$

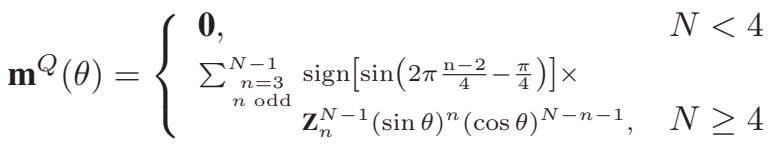

The scalable interplex is an interplex in which the IM terms are scaled:

$$
\begin{aligned}
\mathbf{x}_{N}^{\text {scal }}(\theta, \kappa) & =\mathbf{s}_{1}(\cos \theta)^{N-1}+\kappa^{I} \mathbf{m}^{I}(\theta) \\
& +j\left(-\sum_{n=2}^{N} \mathbf{s}_{n}(\cos \theta)^{N-2} \sin \theta+\kappa^{Q} \mathbf{m}^{Q}(\theta)\right)
\end{aligned}
$$

In this paper we assume $\kappa^{I}=\kappa^{Q}=\kappa$. The scaling factor $\kappa$ is a real number between 0 and 1 . The IM power is given by the sum of the squares of all the terms present in $\mathbf{m}^{I}(\theta)$ and $\mathbf{m}^{Q}(\theta)$, if the spreading codes used to spread the signal components are Gold codes [4]. Indeed, if this is the case, the cross-products between IM terms is negligible, because the product between Gold codes is still a Gold code. The IM power is :

$$
P_{I M}(N, \theta)=\kappa^{2} P_{I M}^{s t d}(N, \theta)
$$

with $P_{I M}^{s t d}(N, \theta)$ the IM power of the standard interplex scheme (5) which has the same value of $N$ and $\theta$. The percentages of the total power that are used for the primary and secondary component are respectively:

$$
\begin{aligned}
p_{\text {main }}(\theta, \kappa)= & {[\cos \theta]^{2(N-1)} } \\
& +\frac{1-\kappa^{2}}{N} p_{I M}^{s t d}(N, \theta) \\
p_{\text {sec }}(\theta, \kappa)= & {[\cos \theta]^{2(N-2)}[\sin \theta]^{2} } \\
& \quad+\frac{1-\kappa^{2}}{N} p_{I M}^{s t d}(N, \theta)
\end{aligned}
$$

with $p_{I M}^{s t d}(N, \theta)$ indicating the percentage of power that is used for the IM product in a standard interplex scheme with the same value of $N$ and $\theta$. The term $\frac{1-\kappa^{2}}{N} p_{I M}^{s t d}(N, \theta)$ accounts for the power that is recovered from the IM terms and it is equally divided among the useful signal components.

Allocation of the additional power for the provision of new services

The challenge of a signal design for the provision of additional SBAS services is that the existing aeronautical service must be left untouched. In our model, the existing aeronautical SBAS L1/L5 service is provided by the primary component. The additional services will be delivered by the secondary components. New services can be incorporated only if additional power becomes available, since a bandwidth enlargement is not foreseen. We define the power increment as the amount of new available power with respect to the minimum power required by the legacy service:

$$
P_{\text {incr }}=\frac{P}{P_{\text {legacy }}}
$$


The power of the primary and secondary components as a function of the power increment are

$$
\begin{gathered}
P_{\text {main }}\left(P_{\text {incr }}\right)=P_{\text {incr }} P_{\text {legacy }} P_{\text {main }}(\theta, \kappa) \\
P_{\text {sec }}\left(P_{\text {incr }}\right)=P_{\text {incr }} P_{\text {legacy }} p_{\text {sec }}(\theta, \kappa)
\end{gathered}
$$

The power share among primary and secondary components is organized as follows. Initially, the additional power is allocated only to the secondary components, which for $P_{i n c r}=0$ (i.e. current SBAS signal), do not exist yet. As the power on the secondary components reaches the minimum power of the legacy service $P_{\text {legacy }}$, then the power is uniformly distributed among primary and secondary components. For each value of $P_{i n c r}$, there is a value of $\theta$, which determines the above mentioned power partition, so (13) are dependent on $P_{\text {incr }}$ through $\theta$. The dependency on $P_{\text {incr }}$ of (13) and on $\kappa$ has been omitted in the following, in order to have a lighter notation.

The SNR (Signal-to-Noise Ratio) also needs to include a margin, in case the satellite link suffers from any channel impairment while still achieving the target frame error rate (FER). The useful SNR is the received SNR, that takes into account this margin. The useful SNRs, that correspond to the above mentioned management of the power resource with (13), are:

$$
\begin{aligned}
S N R_{\text {main }} & =\frac{P_{\text {main }} G}{2 B N_{0} I_{\text {main }}} \\
S N R_{\text {sec }} & =\frac{P_{s e c} G}{2 B N_{0} I_{\text {sec }}}
\end{aligned}
$$

where:

$G$ : processing gain of the DS-CDMA transmission,

$I_{\text {main }}$ : SNR margin for the main component,

$I_{s e c}:$ SNR margin for the secondary component,

$N_{0}$ : noise spectral density,

$B$ : one-sided bandwidth of the received signal.

The SNR values that we used in the following section are calculated from the values shown in Table 1 . The values of

\begin{tabular}{|c|c|}
\hline Parameter & Value \\
\hline$P_{\text {legacy }}$ & $-161 \mathrm{dBW}$ \\
$N_{0}$ & $-204 \mathrm{dBW} / \mathrm{Hz}$ \\
$G$ & $33.10 \mathrm{~dB}$ \\
$\mathrm{~B}$ & $1.1 \mathrm{MHz}$ \\
FER & $10^{-4}$ \\
$I_{\text {main }}$ & $10.93 \mathrm{~dB}$ \\
$I_{\text {sec }}$ & $10.93 \mathrm{~dB}$ \\
\hline
\end{tabular}

Table 1. Parameters for L1 SBAS aeronautical service [1]

the parameters in Table 1 have been taken from [1], with the exception of $I_{\text {main }}$ and $I_{s e c}$, which have been assumed on the basis of typical values.

In Figure 1 and Figure 2 the SNR values on the secondary components as function of the power increment are reported for $\kappa=1$ (standard interplex [2]) and $\kappa=0.25$, respectively.

\section{High power amplifier model}

The Saleh model [6] is an established model to describe the nonlinearities of a HPA. In this paper we use an extension of

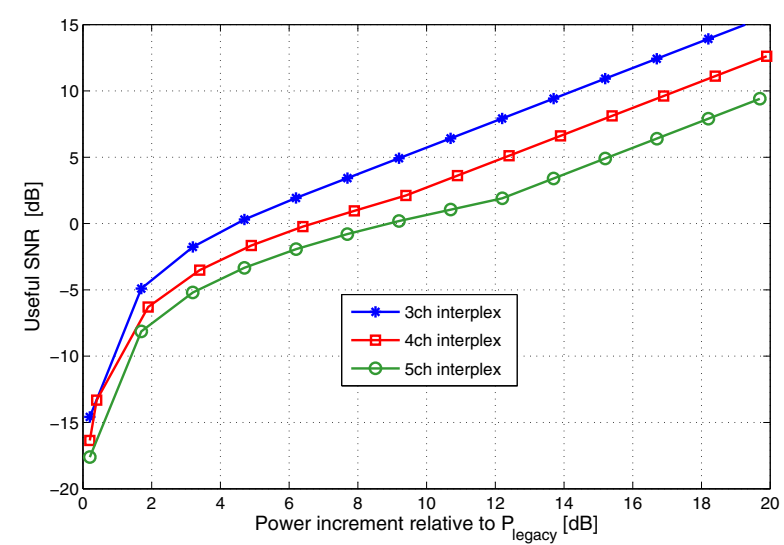

Figure 1. Useful SNR on secondary components for $\kappa=1$.

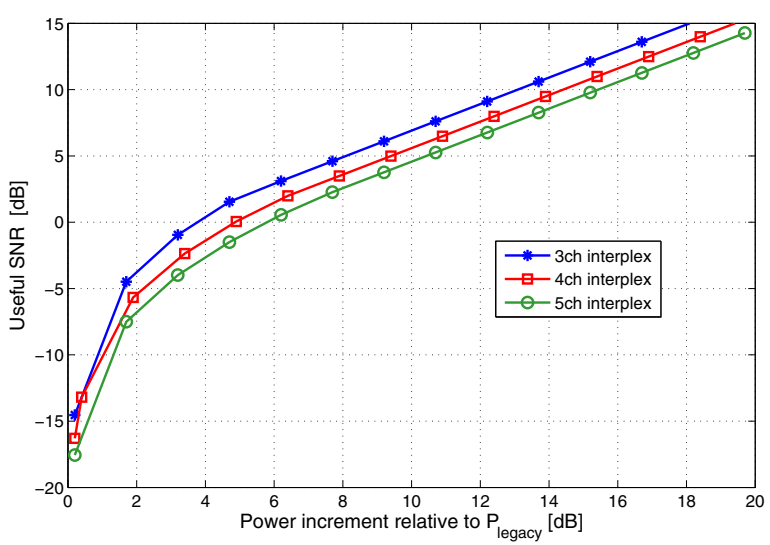

Figure 2. Useful SNR on secondary components for $\kappa=0.25$.

the Saleh model, known in the literature as modified Saleh model [7], [8, p. 113]. The AM-AM characteristic of the modified Saleh model that we use for our assessments is

$$
\tilde{r}_{\text {out }}=\frac{\tilde{r}_{i n}}{1+\tilde{\beta} \tilde{r}_{i n}^{\gamma}}
$$

where $\tilde{r}_{\text {in }}$ and $\tilde{r}_{\text {out }}$ are the input and output signal envelopes respectively, expressed in $\sqrt{\text { Watt }}$. With respect to the original Saleh model [6], this extended model allows to characterize the degree of the nonlinearity for which the HPA is responsible, through the parameter $\gamma$. In the classical model $\gamma=2$. In comparison to the formula in [7], we have ignored any scaling factor of the output as we are only interested in the distortion caused by the HPA. For the other exponents present in [7], we chose the values of the classical AM-AM Saleh model [6]. Moreover, since we are interested in a behavioral analysis, it is handy to write the input envelope as a function of the input saturation power:

$$
P_{\text {sat }}^{i n}=\sqrt[\frac{\gamma}{2}]{\frac{1}{(\gamma-1) \beta}}
$$

The input envelope normalized to the saturation power is

$$
r_{i n}=\frac{\tilde{r}_{i n}}{\sqrt{P_{s a t}^{i n}}}
$$


with

$$
\beta=\tilde{\beta}^{\gamma} \sqrt{W a t t}
$$

Since we are interested only in the distortion and not in the gain brought about by the HPA, we normalize the HPA output to the square root of the power of the input. The power of the input determines the working point of the HPA, indicated by

$$
P_{o p}=E\left[\tilde{r}_{i n}^{2}\right]
$$

The AM-AM characteristic that we consider is such that the HPA does not alter the average signal power:

$$
\bar{r}_{\text {out }}=\frac{r_{\text {in }}}{1+\beta r_{\text {in }}^{\gamma}} \sqrt{\frac{E\left[\tilde{r}_{\text {in }}^{2}\right]}{E\left[r_{\text {out }}^{2}\right]}}
$$

with

$$
r_{\text {out }}=\frac{r_{i n}}{1+\beta r_{i n}^{\gamma}}
$$

where $\bar{r}_{\text {out }}$ is in $\sqrt{\text { Watt }}$ such that it always has the same power of the HPA input $\tilde{r}_{i n}$. This formulation allows to highlight only the power loss caused by the distortion, independently from the HPA gain. We set the working point of the HPA, i.e. the average power of the input signal, at the input saturation power of the AM-AM characteristic:

$$
P_{o p}=E\left[\tilde{r}_{i n}^{2}\right]=P_{s a t}^{i n} \quad \Longrightarrow \quad E\left[r_{i n}^{2}\right]=1 .
$$

This corresponds to an Input power Back-Off (IBO) equal to $0 \mathrm{~dB}$. At this point of the AM-AM curve, both nonlinear distortions and HPA power efficiency are maximal. Furthermore, at this working point the PAPR (Peak-to-AveragePower Ratio) of the interplex constellation has the maximum impact on the power efficiency of the modulation.

For simplicity, in our study we consider an ideal AM-PM curve. The AM-PM curve describes the phase noise that the HPA adds to the amplified signal. If the input signal has a constant envelope, the phase noise is a constant term and it creates no problem at receiver side. If the input signal has a high PAPR, the HPA output is affected by phase noise. A higher phase jitter reduces the power at the output of the receiver's correlator and thus it is also a power inefficiency. Thus, strictly speaking, it would be necessary to compute the increase of the phase jitter of the received signal, for which the HPA is responsible, and then to derive the consequent correlation loss. Nevertheless, in this study we make the approximation that at the working point at which we operate the AM-PM curve is almost constant. If this is the case, the output phase has a limited dependency on the dynamic range of the input envelope, and the phase jitter caused by the HPA can be neglected. This assumption is in agreement with the study of [9], in which the AM-PM curve is almost constant when the input power is equal to the saturation power of the AM-AM curve.

\section{Scalable interplex performance}

In this section we asses the power efficiency of the scalable interplex scheme. The power inefficiencies can be of two kinds: correlation loss due to HPA distortions, and IM power loss. The output of the HPA is

$$
\mathbf{y}=T\left[\mathbf{x}_{N}^{s c a l}\right]
$$

where $\mathbf{x}_{N}^{s c a l}$ indicates the input signal, while $T[\cdot]$ indicated the HPA transfer function described in (21). The signal $\mathbf{y}$ has the same power $P$ as the signal $\mathbf{x}_{N}^{s c a l}$. The transmitted power is partitioned in 3 quotas:

$$
P=\underbrace{P_{u s e}^{R x}+P_{u s e}^{\text {Loss }}}_{=P_{u s e}^{T x}}+P_{I M}
$$

The term $P_{I M}$ is the one in (9). The power $P_{u s e}^{R x}$ is defined as the useful power at the correlator outputs at the receiver and $P_{u s e}^{T x}$ is the useful transmit power. The correlator outputs for the primary and secondary components are respectively:

$$
\begin{aligned}
& z_{1}=\frac{1}{G}<\operatorname{Re}\{\mathbf{y}\}, \mathbf{c}^{1}> \\
& z_{n}=\frac{1}{G}<\operatorname{Im}\{\mathbf{y}\}, \mathbf{c}^{\mathrm{n}}>, \mathrm{n}=2, \ldots, \mathrm{N}
\end{aligned}
$$

where the symbol $<\cdot, \cdot>$ indicates the scalar product between two vectors. The useful received power is

$$
P_{u s e}^{R x}=\sum_{n=1}^{N}\left|z_{n}\right|^{2}
$$

The power loss at receiver side because of HPA distortion is:

$$
P_{\text {use }}^{\text {Loss }}=P_{\text {use }}^{T x}-P_{\text {use }}^{R x},
$$

with

$$
P_{u s e}^{T x}=P_{\text {main }}+(N-1) P_{\text {sec }} .
$$

At this point we can define our metric, which is the total power inefficiency:

$$
\varepsilon=\frac{P_{u s e}^{\text {Loss }}+P_{I M}}{P} .
$$

This quantity is a measure of how much transmitted power cannot be used at receiver side, either because of HPA distortions, or because it was employed in the construction of the IM terms.

We evaluate the metric (30) for two HPAs: one with $\gamma=2$ (classical Saleh model) and another with $\gamma=5$. The former HPA is taken as an example of a typical HPA, and the latter as an example of highly non-linear HPA. Both HPAs are driven at saturation. We performed this evaluation for $P_{2}=P_{1}$. The results are depicted in Figure 3 and Figure 4. In these figures the total power inefficiency of the scalable interplex is compared to the case in which no IM products are transmitted $(\kappa=0)$. This latter case is a special case of the scalable interplex that coincides with a quadrature amplitude modulation (QAM) modulation. The scalable interplex is more power efficient than the QAM when the total power inefficiency falls below the corresponding dashed line, that represents the equivalent QAM scheme. For a better understanding of the trend of the chosen metric with increasing $N$, we decided to show also the case $N=6$. As we can see from Figure 3 , for a typical HPA it is worth while introducing additional power in terms of IM products only for scalable interplex with $N=6$. For smaller values of $N$, the QAM scheme is more power efficient. Nevertheless, for a more non-linear HPA, for each $N$ there exists a value of the IM scaling factor different from zero that minimizes the power inefficiency $\varepsilon$. The advantage of the scalable interplex is that it allows to adapt the signal modulation to the characteristics of the HPA at hand. 


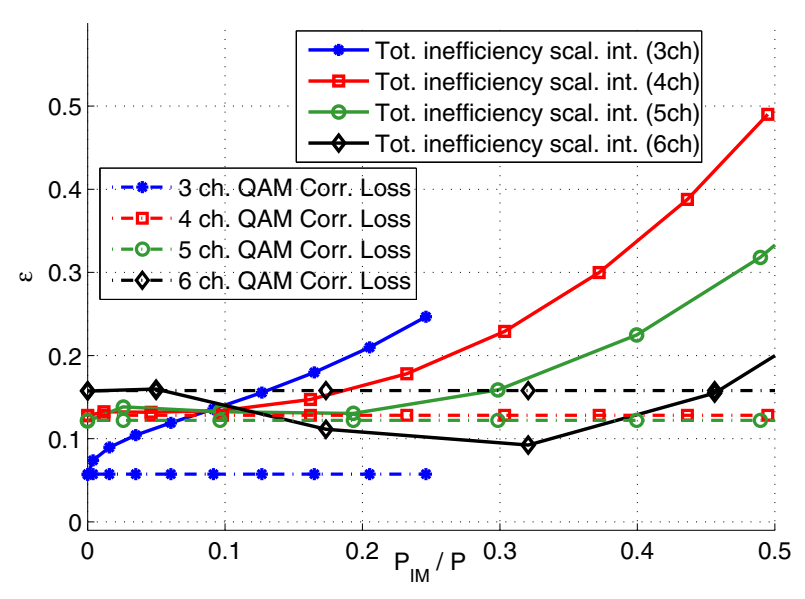

Figure 3. Total power inefficiency calculated for $\frac{P_{s e c}}{P_{\operatorname{main}}}=1$ and a typical HPA $(\gamma=2)$.

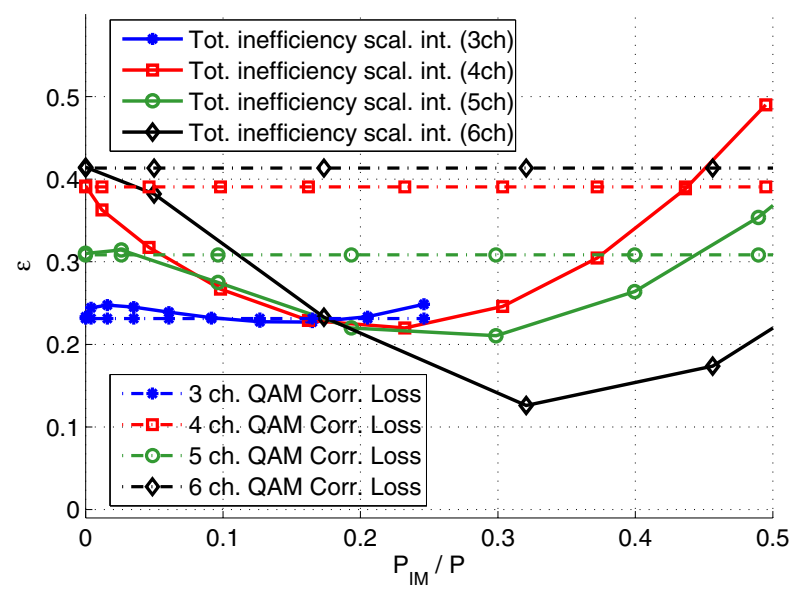

Figure 4. Total power inefficiency calculated for $\frac{P_{s e c}}{P_{\text {main }}}=1$ and a highly non-linear HPA $(\gamma=5)$.

\section{Channel Code Design for the Short BLOCK LENGTH REgIME}

In this section, the potential of channel coding applied to the proposed scheme is investigated. More specifically, we introduce an error correction mechanism which is inspired by multi-level error correcting code [10].

We assume the first $N-1$ signal components to be encoded independently with $(64,57)$ extended Hamming codes. The $N$-th signal component is dedicated to carry additional redundancy computed across the signal components, e.g. the $i$-th bit of the $N$-th is obtained as the sum (over the binary field) of the $i$-th bit of each of the first $N-1$ signal components' codewords. The resulting overall code can be seen as a $(64 \times N, 57 \times(N-1))$ product code [11] given by the serial concatenation of $(64,57)$ extended Hamming codes with $(N, N-1)$ single-parity-check codes. The advantage of this construction resides in the capability of embedding the main component legacy error correcting into a longer block code. This allows to keep the legacy part of the interplex backward compatible. A basic receiver may simply ignore the additional signal components, and perform error correction by using the legacy (e.g. Hamming or convolutional) error correcting code decoder. Enhanced receivers may exploit the additional components not only to retrieve more data, but also to enhance the robustness of the legacy component. In the latter case, decoding is performed iteratively (in a turbo-like fashion) [12-14] by exchanging soft information between the soft-input soft-output decoders of the Hamming and the single-parity-check codes [15].

The proposed code construction may be further generalized, beyond the product construction, towards the class of generalized low-density parity-check (G-LDPC) codes $[3,12,16]$. A G-LDPC code can be conveniently described by a bipartite graph [15] consisting of a set on $n$ variable nodes (one for each codeword bit), $m_{c}$ constraint nodes (one for each subcode). The two sets of nodes are connected in a sparse manner, i.e., there are a few edges connecting variable and constrain nodes. An example of bipartite graph of a G-LDPC code is depicted in Fig. 5. In the figure, the constraint nodes (CNs) are the square nodes marked by $C_{0}, C_{1}, \ldots, C_{m_{c}-1}$ to signify the $m_{c}$ code constraints placed on the code bits associated with the CNs. Variable nodes (VNs) are denoted with circles. The specifications of the subcodes associated with the constraint nodes are also required. The bipartite

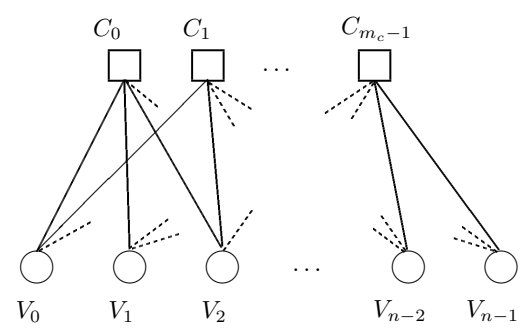

Figure 5. Bipartite graph for a generalized LDPC code.

graph for the proposed construction will then comprise $N$ CNs based on $(64,57)$ extended Hamming codes, $64 \mathrm{CNs}$ based on $(N, N-1)$ single-parity-check codes, and $n=$ $64 \times N$ variable nodes. Each variable node is connected to one $\mathrm{CN}$ of Hamming type and to one $\mathrm{CN}$ of single-parity-check type $[15,17]$.

\section{Numerical results}

Figures 6, 7 and 8 depict the components block error rate (CBER) versus the SNR of the main component, for scaling factors $\frac{P_{s e c}}{P_{\operatorname{main}}} \in\{1,0.75,0.5\}$, respectively. A component block is considered erroneous whenever the component is decoded with errors. For $\frac{P_{s e c}}{P_{\operatorname{main}}}=1$ (Figure 6), the same power is allocated to all the components. Thus, there is no distinction between the error rate achieved over the main and the secondary components. For $\frac{P_{\text {sec }}}{P_{\operatorname{main}}}=0.75$ and $\frac{P_{s e c}}{P_{\operatorname{main}}}=$ 0.5 (Figures 7 and 8), the main component enjoys a power boost with respect to the secondary ones. Accordingly, the error rate measured over the secondary components suffers for a loss with respect to that of the main component, with a gap that increases for lower $\frac{P_{s e c}}{P_{\operatorname{main}}}$. The loss is in fact negligible for $\frac{P_{s e c}}{P_{\text {main }}}=0.75$, whereas the difference is evident for $\frac{P_{\text {sec }}}{P_{\text {main }}}=0.5$. Note that, since the plots are provided as a function of the SNR of the main component, the schemes employing more components take advantage of a higher overall power. 


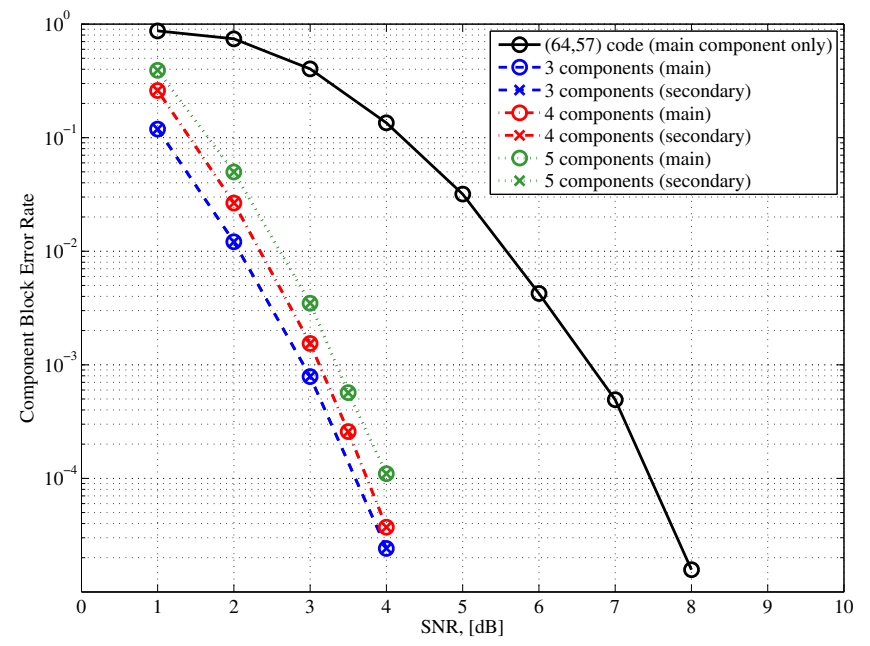

Figure 6. Block Error Rate per component as a function of the SNR on the main component. $\frac{P_{s e c}}{P_{\operatorname{main}}}=1$.

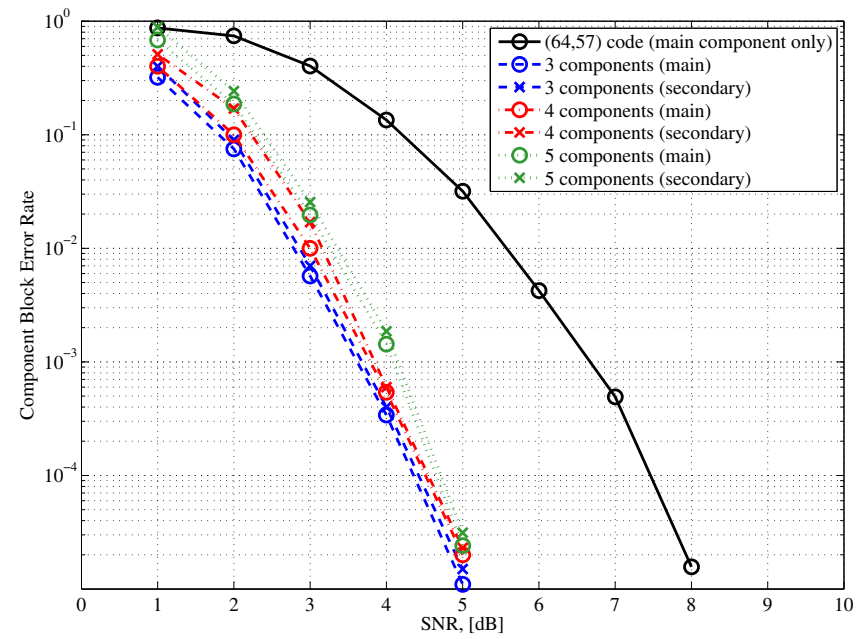

Figure 7. Block Error Rate per component as a function of the SNR on the main component. $\frac{P_{s e c}}{P_{\operatorname{main}}}=0.75$.

\section{Conclusions}

In this work we have proposed a novel interplex scheme which provides the capability of shaping the signal constellation allowing to adapt the signal to the available HPA in order to achieve low total power inefficiency.

Furthermore, we have proposed an innovative coding scheme that encodes all signal components in a joint fashion developing G-LDPC codes. This framework allows jointly encoding and decoding the legacy signal and the additional components. Simultaneously, the baseline code that is protecting the legacy signal is embedded in the overall coding scheme. In this work we have presented an example of such a coding scheme based on extended Hamming codes instead of convolutional codes as defined in [1].

Applying both the novel scalable interplex scheme and the proposed innovative coding scheme the available power can efficiently be used to increase the overall data rate for a multi-service SBAS without changing the legacy signal. The

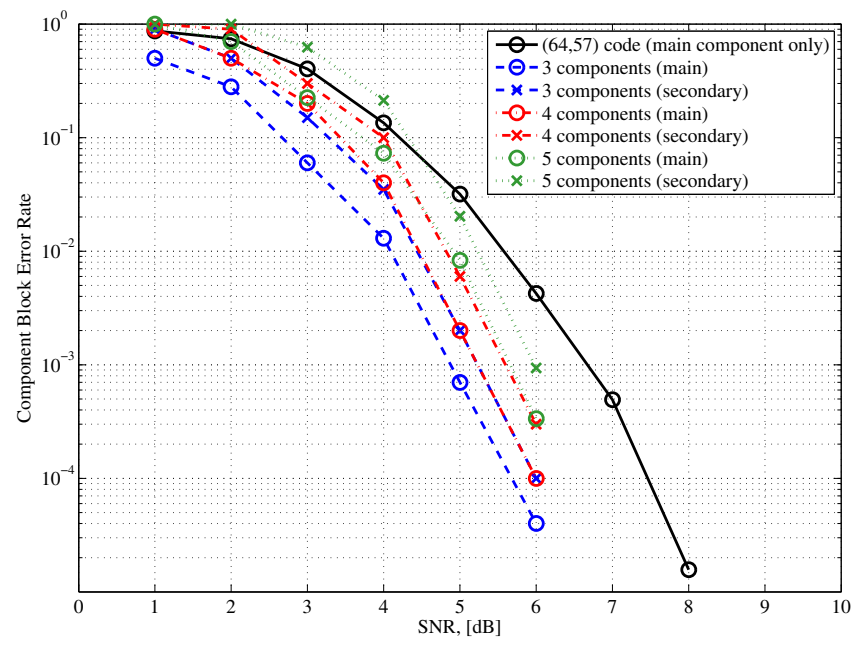

Figure 8. Block Error Rate per component as a function of the SNR on the main component. $\frac{P_{s e c}}{P_{\operatorname{main}}}=0.5$.

proposed approach can also be applied for evolution or modernization of GNSS (Global Navigation Satellite Systems) in order to achieve backward compatibility of signals and services.

\section{REFERENCES}

[1] "Signal Specification for SBAS L1/L5," EuroCAE ED134, May 2008, Draft v.3.

[2] S.Butman and Uzi Timor, "Interplex an efficient multichannel psk/pm telemetry system," IEEE Trans. on Communications, vol. 20, no. 3, pp. 415-419, June 1972.

[3] G Liva, W E Ryan, and M Chiani, "Quasi-cyclic generalized ldpc codes with low error floors," IEEE Transaction on Communications, vol. 56, pp. 47-57, January 2008.

[4] R. Gold, "Optimal binary sequences for spread spectrum multiplexing (corresp.)," IEEE Transactions on Information Theory, vol. 13, no. 4, pp. 619-621, October 1967.

[5] M. Vergara and F. Antreich, "Staggered Interplex," in IEEE/ION PLANS 2012, Myrtle Beach, SC, USA, April 2012.

[6] A. Saleh, "Frequency-independent and frequencydependent nonlinear models of twt amplifiers," IEEE Trans. on Communications, vol. 29, no. 11, November 1981.

[7] M. O'Droma, “New modified saleh models for memoryless nonlinear power amplifier behavioural modelling," IEEE Communications Letters, vol. 13, no. 16, pp. 399-401, July 2009.

[8] D. Schreurs, M. O'Droma, A. A. Goacher, and M. Gadringer, RF Power Amplifier Behavioral Modeling, Cambridge university press, 2009.

[9] A.R. Kaye, D.A. George, and M.J. Eric, "Analysis and compensation of bandpass nonlinearities for communications," IEEE Transactions on Communications, vol. 20, no. 5, pp. 965-972, October 1972.

[10] H. Imai and S. Hirakawa, "A new multilevel coding 
method using error-correcting codes," IEEE Transactions on Information Theory, vol. 23, no. 3, pp. 371 377, may 1977.

[11] P. Elias, "Error-free coding," IRE Trans. Inf. Theory, vol. PGIT-4, pp. 29-37, Sept. 1954.

[12] R. G. Gallager, Low-Density Parity-Check Codes, M.I.T. Press, Cambridge, MA, 1963.

[13] C. Berrou, A. Glavieux, and P. Thitimajshima, "Near Shannon limit error-correcting coding and decoding: Turbo-codes," in Proc. IEEE International Conference on Communications, Geneva, Switzerland, May 1993.

[14] R. Pyndiah, "Near optimum decoding of product codes: Block turbo codes," IEEE Transactions on Communications, vol. 46, no. 8, pp. 1003-1010, Aug. 1998.

[15] R.M. Tanner, "A recursive approach to low complexity codes," IEEE Transactions on Information Theory, vol. 27, no. 5, pp. 533-547, Sept. 1981.

[16] M. Lentmaier and K. S. Zigangirov, "Iterative decoding of generalized low-density parity-check codes," in Proc. IEEE International Symposium on Information Theory, Boston, USA, Aug. 1998.

[17] M. Lentmaier, G. Liva, E. Paolini, and G. Fettweis, "From Product Codes to Structured Generalized LDPC Codes," in Proc. Chinacom 2010, Beijing, China, Aug. 2010.

\section{BIOGRAPHY}

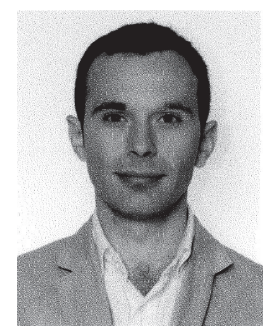

Mariano Vergara received the BSc. and the MSc. degrees in Telecommunications engineering from the University of Naples Federico II,Italy, in 2006, and from the Technische Universität Kaiserslautern (TU-KL), Germany, in 2009, respectively. He is currently pursuing a Ph.D. degree at the Universtat Autònoma de Barcelona (UAB), Spain, on signal design for global navigation satellite systems (GNSS). Since 2008, he has been an Associate Researcher with the Department of Navigation, Institute of Communications and Navigation of the German Aerospace Center (DLR).

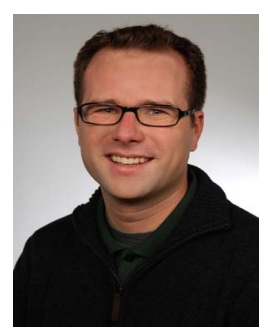

Felix Antreich (M'06) was born in $\mathrm{Mu}-$ nich, Germany, in 1977. He received the diploma and the Ph.D. degree in electrical engineering from the Technische Universität München (TUM), Germany in 2003 and 2011, respectively. Since July 2003, he has been an Associate Researcher with the Department of Navigation, Institute of Communications and Navigation of the German Aerospace Center (DLR). His research interests include sensor array signal processing for global navigation satellite systems (GNSS) and wireless communications, estimation theory and signal design for synchronization, and GNSS. He serves IEEE as reviewer for Transactions, Journals, and Conferences.

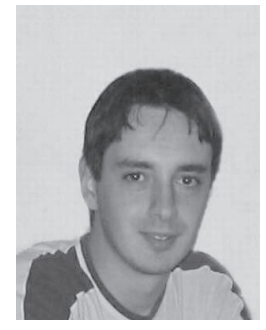

Gianluigi Liva was born in Spilimbergo, Italy, on July 23rd, 1977. He received the M.S. and the Ph.D. degrees in electrical engineering from the University of Bologna (Italy) in 2002 and 2006, respectively. His main research interests include satellite communication systems, random access techniques and error control coding. Since 2003 he has been involved in the research of channel codes for high data rate CCSDS (Consultative Committee for Space Data Systems) missions, in collaboration with the European Space Operations Centre of the European Space Agency (ESA-ESOC). From October 2004 to April 2005 he was researching at the University of Arizona as visiting student, where he was involved in the design of lowcomplexity coding systems for space communication systems. He is currently with the Institute of Communications and Navigation at the German Aerospace Center (DLR). He is / he has been active in the DVB-SH, DVB-S2 and in the DVB-RCS standardization groups. In 2010 he has been appointed guest lecturer for channel coding at the Institute for Communications Engineering (LNT) of the Technische Universität München (TUM). In 2012 he has been lecturing for channel coding at the Nanjing University of Science and Technology in Changshu (China). He is IEEE member and he serves IEEE as reviewer for Transactions, Journals and Conferences.

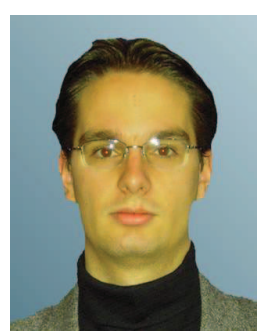

Balazs Matuz was born in Budapest, Hungary, in 1982. He received his Diploma degree in Electrical Engineering and Information Technology from the Technische Universität München (TUM), Germany in 2007. Since then he is enrolled in a $\mathrm{PhD}$ program at the German Aerospace Center (DLR) in Oberpfaffenhofen, Germany. Currently he is a scientific researcher at the Institute of Communications and Navigation where he mainly works on novel forward error correcting schemes (based on LPDC and turbo-like codes) for satellite and space systems. During the past years he has been involved in several national and international projects on channel coding and channel modeling. Amongst others he participated in development of a DVBSH prototype system in the framework of the European Space Agency (ESA) financed projects Ortigia and J-Ortigia. He is an IEEE student member and he serves IEEE as a reviewer for Transactions, Journals and Conferences, as well as a technical program committee member. 